\title{
Murine Embryonic Stem Cells Synthesize Retinoic Acid to Promote their Own Differentiation
}

Francesco Neri" ${ }^{2 \#}$ Caterina De Clemente ${ }^{1,3 \#}$, Maurizio Orlandini ${ }^{1}$, Claudia Lentucci ${ }^{1,4}$, Francesca Anselmi ${ }^{1}$ and Federico Galvagni ${ }^{1 *}$

${ }^{1}$ Dipartimento di Biotecnologie, Chimica e Farmacia, Università di Siena, via A. Moro, 2, 53100 Siena, Italy

${ }^{2}$ HUGEF, via Nizza 52, Torino, Italy

${ }^{3}$ Dipartimento di Scienze Mediche, Chirurgiche e Neuroscienze, Università di Siena, Viale M. Bracci, 16, 53100 Siena, Italy

${ }^{4}$ Biochemistry Department, Boston University School of Medicine, 72 E. Concord St-Boston, MA 02118, USA

"Francesco Neri and Caterina De Clemente contributed equally to this work

\begin{abstract}
Objective: Embryonic stem cells (ESC) pluripotency is maintained through a complex interplay of different signaling pathways and a network of transcription factors. Although much is known about this pluripotency selfrenewal circuitry, the molecular events that lead ESC to exit from pluripotency and begin differentiation are currently less known. All-trans retinoic acid (atRA), plays important and pleiotropic roles in embryonic development and ESC differentiation. The objective of the present study is to check the autocrine synthesis of atRA by ESC, test its role in spontaneous differentiation of ESC as embryoid bodies (EBs), and analyze the expression of the enzymes and proteins involved in atRA synthesis pathway.
\end{abstract}

Methods: ESC, undifferentiated or differentiating as EBs, were grown in absence or presence of atRA, retinol, or the RAR antagonist CD2665, and Brachyury expression was analyzed as marker of ESC differentiation state. ESC or EBs-conditioned medium was produced in absence or presence of retinol and tested on RARE-luciferase reporter cells. RT-qPCR analysis of atRA biosynthetic pathway components was performed on undifferentiated or differentiating ESC. Finally, microarray gene expression profile was used to identify direct atRA target genes in ESC.

Results: Here, we demonstrate that atRA promotes early steps of ESC differentiation, and that ESC increase their capacity to synthesize atRA during spontaneous differentiation as EBs, up-regulating RDH1, RDH10, ADH3, RALDH2, and CRABP2. Among 35 transcription factors (TFs) regulated by atRA in ESC, 3 TFs up-regulated (Snai1, Gata6, Cdx1) are known to be involved in ESC pluripotency exit and 3 TFs down-regulated (Otx2, Id2 and Arid1a) are involved in ESC pluripotency maintaining.

Conclusion: Cultivation and controlled differentiation of ESC has opened new frontiers both in regenerative medicine and biology of development. Here, we demonstrated that RA is synthesized by ESC during spontaneous differentiation as EBs and takes an active role to promote their own differentiation process.

Keywords: Retinoic acid; Retinol; Embryonic stem cells; Differentiation; Self-renewal; RDH1; RDH10; ADH3; RALDH2; CRABP2

\section{Introduction}

The first cell lineage specification in mammalian embryo development is the formation of trophectoderm (TE) and inner cell mass (ICM) of the blastocyst. TE cells will form a large part of the placenta, whereas ICM will give rise to the epiblast and primitive endoderm. After embryo implantation the epiblast generates the three germ layers, and hence all the tissues in the future body. ESC are derived from blastocyst-stage embryos and are thought to be functionally equivalent to the ICM.

Vitamin A (retinol) is obtained from the diet in the form of retinyl ester and oxidized in a two-step process, first to all-trans retinaldehyde and then, in irreversible fashion, to atRA. The first step of atRA synthesis is catalyzed by several members of two enzyme families: the alcohol dehydrogenases (ADHs) and retinol dehydrogenases (RDHs). Retinaldehyde is then further converted to retinoic acid by retinal dehydrogenases (RALDH). RA crosses the plasma membrane and is bound by cellular retinoic acid binding protein CRABP-I or CRABPII. atRA acts within the nucleus as a ligand to two nuclear receptor families (RAR and RXR), which regulate transcription of target genes by binding RA response elements (RARE) [1]. In placental embryos, autocrine retinoic acid synthesis begins at 7.5 d.p.c. and the major source of retinoids is maternal retinol [2]. atRA plays important and pleiotropic roles in embryo development and cellular differentiation [3] and prolonged atRA exposure promotes ESC differentiation into primitive endoderm or cells of the three primary germ layers, depending on culture conditions [4-6].

Recently, we identified Snail as direct target of retinoid receptors and found that Snail promotes ESCs differentiation into primitive endoderm and cells of the three primary germ layers through the direct binding and repression of pluripotency and self-renewal genes such as Nanog, Tcfcp2l1 and Nr5a2 [7]. Here we demonstrate that atRA promotes early steps of ESC differentiation, and that ESC increase their capacity to synthesize atRA during spontaneous differentiation as embryoid bodies, up-regulating the atRA biosynthetic pathway components RDH1, RDH10, ADH3, RALDH2, and CRABP2.

*Corresponding author: Federico Galvagni, Dipartimento di Biotecnologie Chimica e Farmacia, Università di Siena, via A. Moro, 2, 53100 Siena, Italy, Tel: +39 0577 234961; Fax:+39 0577 234254; E-mail: federico.galvagni@unisi.it

Received June 15, 2015; Accepted July 21, 2015; Published July 23, 2015

Citation: Neri F, De Clemente C, Orlandini M, Lentucci C, Anselmi F, et al. (2015) Murine Embryonic Stem Cells Synthesize Retinoic Acid to Promote their Own Differentiation. J Stem Cell Res Ther 5: 293. doi:10.4172/2157-7633.1000293

Copyright: (c) 2015 Neri F, et al This is an open-access article distributed under the terms of the Creative Commons Attribution License, which permits unrestricted use, distribution, and reproduction in any medium, provided the original author and source are credited. 


\section{Materials and Methods}

\section{ESC maintenance}

Mouse E14 ESC were cultured as previously described [8] on gelatin-coated dishes in feeders free culture system DMEM $(4.5$ $\mathrm{g} / \mathrm{l}$ glucose) containing 15\% Embryomax fetal bovine serum (FBS, Millipore), $1 \mathrm{mM}$ sodium pyruvate (Invitrogen), $0.1 \mathrm{mM}$ MEM nonessential amino acid (Invitrogen), $1000 \mathrm{U} / \mathrm{ml}$ LIF ESGRO (Millipore), and $50 \mu \mathrm{M} 2$-Mercaptoethanol. Mouse GFP-Bry ES cell line was kindly provided by Dr. Gordon Keller, and cultured in serum-free medium on gelatin-coated dishes in feeders free culture system as previously described [9]. ESC-RARE-LUC cells were cultured and differentiated as wt E14 ESC.

\section{ESC differentiation}

For EBs formation, E14 ESC were plated at $2.5 \times 10^{5}$ cells $/ \mathrm{ml}$ in ultra low attachment plates (Costar) in MEM ALPHA Medium (Invitrogen) supplemented with $10 \%$ FBS or Charcoal/Dextran Treated FBS (HyClone) and $50 \mu \mathrm{M}$ 2-Mercaptoethanol. After 48 hours EBs were treated with $1 \mu \mathrm{M}$ CD2665 (R\&D Systems) or $0.1 \mu \mathrm{M}$ Retinol (\#R7632, Sigma-Aldrich). GFP-Bry derived EBs were cultured in serum-free medium consisting of 75\% IMDM (Invitrogen), 25\% Ham's F12 (Invitrogen) supplemented with 0.5x N2B27 (w/o retinoic acid) supplement (Invitrogen), 0.05\% BSA, $2 \mathrm{mM}$ glutamine (Invitrogen), 0.5 $\mathrm{mM}$ ascorbic acid (Sigma-Aldrich), $0.45 \mathrm{mM}$ 1-thioglycerol (Sigma-
Aldrich). After 24 hours GFP-Bry EBs were treated for additional 24 hours with $0.1 \mu \mathrm{M}$ atRA (\#R2625, Sigma-Aldrich). Subsequently, EBs were dissociated with trypsin and cells were reaggregated at $1.5 \times 10^{5} \mathrm{cells} /$ $\mathrm{ml}$ in the same medium and new EBs were harvested 3 days later for flow cytometry analysis.

\section{Luciferase assay}

Lentiviral vectors pGREENFIRE1-mCMV-EF1-PURO (for background control) and pGREENFIRE1-RARE-mCMV-EF1-PURO (System Bioscience) were used to produce lentiviral particles as previously described [10]. E14-ESC were transduced with lentiviral particles and 24 hours after infection medium was changed and cells treated with Puromycin $6 \mu \mathrm{g} / \mathrm{ml}$ for 5 days. Selected cells were cultured for 8 hours in the indicated media, lysed and luciferase activity was measured by using the Dual-Luciferase ${ }^{\oplus}$ Reporter Assay (Promega) as described in [11].

\section{RT-qPCR}

Total RNA was extracted using TRIreagent (Invitrogen) according to the manufacturer's protocol. RT-qPCR was performed as previously described [12] using SuperScript III One-Step RT-PCR System and SYTO9 Green-Fluorescent Nucleic Acid Stain (Invitrogen) on Rotor Gene 6000 (Corbett Research). The primers used for RT-qPCR were designed using Primer3 software from a recent E14 genome assembly [13]. The oligonucleotides used are listed in Table 1.

\begin{tabular}{|c|c|c|}
\hline GENE & Forward sequence $\left(5^{\prime} \rightarrow 3\right)^{\prime}$ & Reverse sequence $\left(5^{\prime} \rightarrow 3\right)$ \\
\hline$\beta$-Actin & TCTTTGCAGCTCCTTCGTTG & ACGATGGAGGGGAATACAGC \\
\hline Brachyury & СTGTGACTGCCTACCAGAATGAGGAG & GGTCGTTTCTTTCTTTGGCATCAAG \\
\hline Rdh1 & CATCTGACAGGCTGGAGACA & AGTCCTCTGTTCCCAACACG \\
\hline Rdh10 & AAGGCCTTTCTTCCAACGAT & CAAGGTAAGGGCAAACCAGA \\
\hline Adh1 & GTTGAGAGCGTTGGAGAAGG & TCGCTTCGGCTACAAAAGTT \\
\hline Adh3 & GTGGGAGTAGCTGCTTCAGG & TGCATCAGATCAAAGGCTTG \\
\hline Adh2 & AAAGCATCCGAACAATCCTG & AGGCTTTCTTTGGCTCATCA \\
\hline Raldh1 & CTCCTGGCGTGGTAAACATT & CCATGGTGTGCAAACTCAAC \\
\hline Raldh2 & TTGCAGATGCTGACTTGGAC & TCTGAGGACCCTGCTCAGTT \\
\hline Raldh3 & GTGGAGTTCGCCAAGAAGAG & AGAAGACCGTGGGTTTGATG \\
\hline Crbp1 & CTTACTGGACCCGAGAGCTG & GGAAAATGGGGTTGCCTAAT \\
\hline Crbp2 & GGTGAAATGGGAGAGTGGAA & GGAAGTCGTCTCAGGCAGTT \\
\hline Crabp1 & CAGTGTGTGCAGAAGGGAGA & AGGTTATCTCCTCGGGCTGT \\
\hline Crabp2 & TAGTCTCCCTGCAGCCTGTT & TGCTGTTGGTTTTCGTCTTG \\
\hline Nanog & CAGGTGTTTGAGGGTAGCTC & CGGTTCATCATGGTACAGTC \\
\hline Oct3/4 & GGCGTTCTCTTTGGAAAGGT & CTTCGGGCACTTCAGAAACA \\
\hline Snai1 & TTGGGCCAACTTCCCAAGCA & AGAGAAGGCCTTTCCACAGGT \\
\hline Aebp2 & ATTGGGACATCGGGACAGCATA & TGGTCCCAGCAACAGTTATAGGC \\
\hline $\mathrm{Cdx1}$ & AAGGACCTGTTCTGAGTTGC & TCGTTGAGCCCTCTGAATCT \\
\hline Сур26a1 & TGCAGCTCCTGATTGAGCACT & TCGCAGCACTGGCTGTAGTTT \\
\hline Foxa1 & AGACATTCAAGCGCAGCTAC & TGCTGGTTCTGGCGGTAATA \\
\hline Foxn4 & AACCTGGCTCGCATCGACAA & TTTCCGGGCGGTCTGAGATGA \\
\hline Gata6 & AAAACGCCAACCCCGAGAA & AGTGGTATGAGGCCTTCAGA \\
\hline Otx2 & TGGAACAAGTGGCCAGTTCA & AAGTGGACAAGGGGTCAGACA \\
\hline Arid1a & TCAGCCTGTGTTGAAGCAGA & TGGCTGAGGTTGAAGGTCAT \\
\hline Neurog3 & CGGATGACGCCAAACTTACA & TAGAAGCTGTGGTCCGCTAT \\
\hline Meox1 & AACCCCGTTTTCCTTCCA & CTGCTCGTTGAAGATTCGC \\
\hline Id2 & TCCAAGCTCAAGGAACTGGT & TGATGCAGGCTGACGATAGT \\
\hline Mist1 & TGCGCCAGGCCCTAAATTAT & TCCTCAGTGACACCAAGCAT \\
\hline Hoxa1 & AGTTGTGGTCCAAGCTATGGC & ACCATGGGAGTCGAGAGGTTTT \\
\hline Hoxa5 & CGAGCCACAAATCAAGCACACA & TCGCTCACGGAACTATGATCTCCA \\
\hline Hoxb4 & AAGTTCACGTGAGCACGGTA & GCGTCAGGTAGCGATTGTAGT \\
\hline Rarb & AGCACCGGCATACTGCTCAAT & TTGTCCTGGCAAACGAAGCA \\
\hline
\end{tabular}

Table 1: Primers sets used for RT-qPCR. 


\section{Flow cytometry analysis}

EBs generated from GFP-Bry cell differentiation were treated with Dissociation Buffer PBS-based (Invitrogen) for 1-2 min and arranged at $1 \times 10^{6}$ cells $/ \mathrm{ml}$ in PBS. $1 \mu \mathrm{l}$ of reconstituted fluorescence reactive dye LIVE/DEAD Fixable Dead Cell Stain Kit (Invitrogen) was added and cells were incubated for $30 \mathrm{~min}$ protecting from light and analyzed by FACS CantoII (Becton Dickenson). Data analysis was performed by FlowJo software (Tree Star Inc.).

\section{Microarray analysis}

Microarray was performed on Illumina Platform and analyzed using BeadStudio Gene Expression Module (GX). Data were background adjusted and quantile normalized using default parameters in the BeadStudio Software. Probes with Log $|\mathrm{FC}|>1+$ and $\mathrm{p}$-value $<0.05$ were selected for downstream analysis. Heat-map plots were performed through the Bioconductor package in R. Differential expression analysis of the up- or down-regulated genes was performed by plotting genes on their $\log _{2}$ expression value using Excel (Microsoft ${ }^{\mathrm{TM}}$ ).

\section{Results and Discussion}

\section{RA promotes ESC spontaneous differentiation}

ESC have the ability to spontaneously differentiate and exogenous RA promotes their differentiation into primitive endoderm and a broad spectrum of derivatives of all three germ layers depending on culture conditions [4-6].

We used EBs as a model system recapitulating the embryogenesis to explore the early steps of RA signaling during embryonic development. For this set of experiments, we used the ES cell line with the green fluorescent protein (GFP) cDNA targeted to the primitive streak marker Brachyury locus (GFP-Bry), which does not differentiate in serum-free medium in absence of specific factors $[9,14,15]$. GFP-Bry ESC were differentiated as EBs in serum-free medium, and after 24 hours, when cells begin the transition from an ICM-like cells to an epiblast-like stem cell (EpiSC) [16], EBs were treated with a 24 hour pulse of atRA. At the end of day 2 EBs were dissociated to eliminate any trace of atRA and reaggregated in differentiation medium without atRA. Differentiation potential was evaluated by flow cytometry at day 5 (Figure 1A). In our experience, the EBs dissociation and reaggregation delayed differentiation timing and day 5 corresponded to day 3 of the original protocol without dissociation [14]. The pulse-type application of atRA induced a large GFP-Bry population compared to untreated cells (Figure 1B), demonstrating that atRA can act early in ESC differentiation, probably promoting loss of self-renewal, in agreement with the observations that atRA represses the expression of the pluripotency genes through direct activation of the transcriptional repressor Snail [7].

To evaluate the influence of RA pathway on spontaneous ESC differentiation in serum-containing medium, cells were allowed to differentiate as EBs, and treated from the end of day one with CD2665, a RAR antagonist [17], or vehicle alone (DMSO). mRNA was collected at the end of day 3 and 4, and Brachyury (Bry) expression was analyzed as marker of early ESC differentiation state. In CD2665 treated cells Bry expression is down-modulated compared to control cells (Figure 1C), supporting the hypothesis that RA is involved in spontaneous ESC differentiation.

\section{ESC increase their capacity to produce atRA during spontaneous differentiation as EBs}

Because key components of the RA synthesis pathway are expressed
A

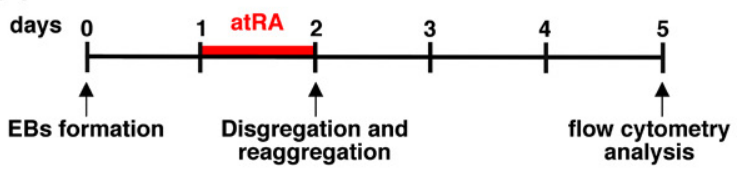

B
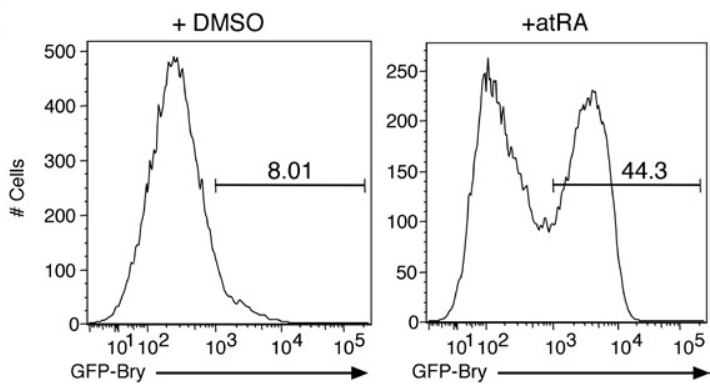

C

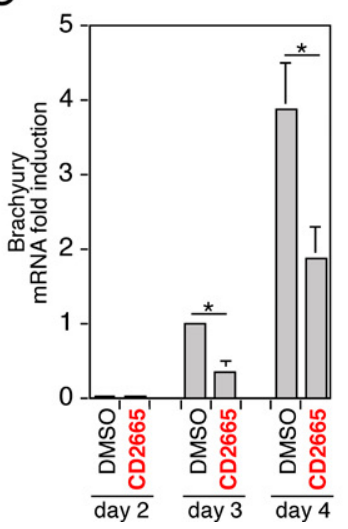

Figure 1: RA promotes ESC spontaneous differentiation. (A) Schematic representation of the experimental procedure used to test the ESC differentiation potential after a single pulse stimulation by RA. (B) FACS profiles of the atRA and DMSO treated cells analyzed for expression of GFP at day 5 . Data are representative of three independent experiments. (C) Spontaneous differentiation of ESC as EBs is down-regulated by the RAR antagonist CD2665. ESC were let to differentiate spontaneously as embryoid bodies for 2, 3 or 4 days, in presence of $1 \mu \mathrm{M}$ CD2665 or vehicle alone (DMSO). Differentiation was evaluated by quantitative reverse transcription-polymerase chain reaction (RT-qPCR) analysis of the early differentiation marker Brachyury. Values are normalized to $\beta$-Actin expression and represented as fold change compared with DMSO-treated EBs at day 3 after formation. Data represent average $\pm S D$ of three independent experiments. ${ }^{*}, P<0.05(n=3)$.

at very low mRNA levels, Simandi et al. suggested that endogenous production of RA from serum-contained retinol is unlikely in undifferentiated ESC [18]. To verify this hypothesis and to assess the ESC ability to synthesize RA during spontaneous differentiation as EBs, we created a stable clone of ESC (ESC-RARE-Luc) carrying the Luciferase reporter gene under the control of RARE. ESC-RARE-Luc were cultured for 8 hours in growth medium conditioned by 24 hours culture of ESC or EBs at different times after LIF withdrawal. As shown in Figure 2A, ESC conditioned medium (ESC+LIF) does not induce significant increase of Luciferase signal with respect to unconditioned medium ( $\mathrm{p}=0.157)$. To overcome the difficulty to estimate the retinol level in serum, we treated ESC-RARE-Luc with medium conditioned by ESC cultured in the presence of exogenously added retinol. In these conditions, a significant increase of Luciferase signal was detected $(0.1$ $\mu \mathrm{M}$ retinol, $\mathrm{p}=0.002 ; 1 \mu \mathrm{M}$ retinol, $\mathrm{p}=0.018)$. The Luciferase signal 
A
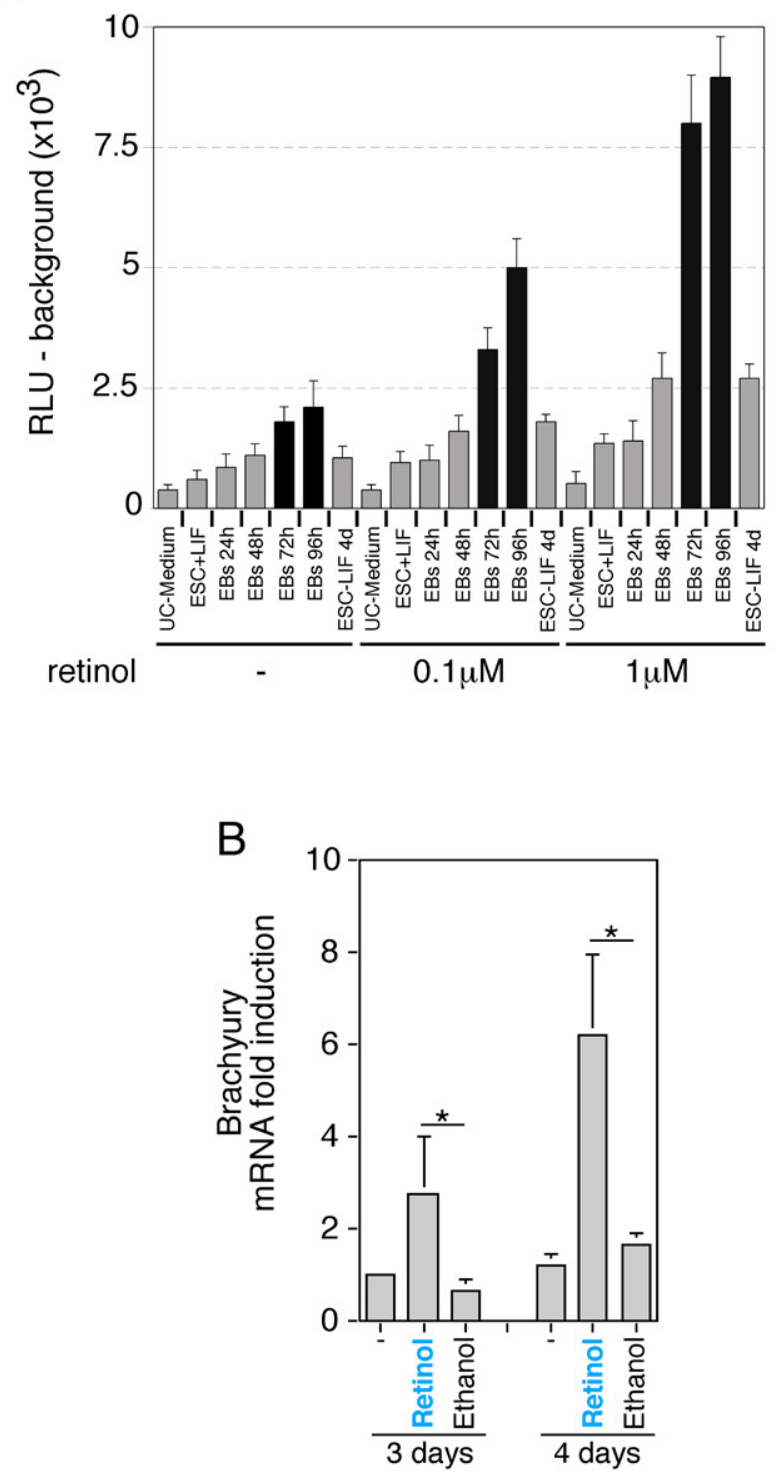

Figure 2: Undifferentiated ESC produce low levels of RA, and increase this capacity during spontaneous differentiation as EBs. (A) A stable clone of ESC (ESC-RARE-Luc) carrying the Luciferase reporter gene under the control of retinoic acid response elements (RARE) was treated for 8 hours with conditioned medium from undifferentiated ESC (ESC+LIF) or EBs at different stages of differentiation. Where indicated, retinol was added to medium during conditioning. UC: unconditioned medium. Relative Light Unit (RLU) values are represented after background subtraction. Background was measured using wild type E14 ESC treated with UC-medium. Data represent average $\pm S D$ of three independent experiments. (B) Retinol supplementation increases spontaneous EBs differentiation in medium supplemented with Charcoal/ Dextran treated FBS. Differentiation was evaluated by RT-qPCR analysis of Brachyury. Values are normalized to $\beta$-Actin expression and represented as fold change compared with untreated EBs at day 3 after formation. Vehicle alone (Ethanol) was used as a control. Data represent average $\pm S D$ of three independent experiments. ${ }^{*}, \mathrm{P}<0.05(\mathrm{n}=3)$.

gradually increased treating ESC-RARE-Luc with medium conditioned by culture of EBs at different times after LIF withdrawal. This increase is much less pronounced with conditioned medium obtained by culture of ESC as monolayer in absence of LIF for 4 days. These data demonstrate that undifferentiated ESC produce RA from retinol, even if at low levels, and the synthesis of RA increases during their spontaneous differentiation into EBs.

To further confirm that ESC can synthesize RA from retinol and use it to promote their own differentiation in serum-containing medium, we differentiated E14 ESC as EBs in medium containing Charcoal/ Dextran absorbed serum to deplete the basal levels of retinoids. After 24 hours, $1 \mu \mathrm{M}$ retinol was added, and RNA was extracted at 2 and 3 days later (corresponding to 72 and 96 hours of EBs differentiation). Retinol treatment enhances ESC differentiation evaluated by RT-qPCR for Bry expression (Figure 2B).

\section{Expression of atRA biosynthetic pathway components during spontaneous ESC differentiation}

Since we observed an increase of RA synthesis in differentiating EBs, we wondered whether the expression of the RA biosynthetic pathway enzymes and retinoid transport proteins could be correlated with this finding. Thus, we quantified the mRNA levels of the enzymes that catalyze retinol oxidation to retinal (RDH1, RDH10 ADH1, ADH3 and $\mathrm{ADH} 4$, for the ADHs nomenclature used see [19]) and retinal oxidation to atRA (RALDH1, 2 and 3), and for retinol (CRBP1 and 2) and RA (CRABP1 and 2) binding proteins (Figure 3A). As reference standards, we used RNA from primary mouse embryonic fibroblasts (MEF), mouse embryo fibroblast cell line NIH3T3 (3T3), and mouse liver. $\mathrm{RDH} 1, \mathrm{ADH} 1$ and $\mathrm{ADH} 4$ are undetectable in ESC and, among them only RDH1 significantly increases after 72 and 96 hours of EBs differentiation. RDH10 and AHD3 are the most expressed retinol dehydrogenases in undifferentiated ESC, and their expression increases during differentiation, especially for $\mathrm{RDH} 1$ and $\mathrm{ADH} 3$ (Figure 3B).

Our findings are consistent with the expression of these enzymes during embryonic development, where $\mathrm{ADH} 3$ is detected in mouse pre-implantation embryo [20], and RDH10 and RDH1 in early postimplantation embryo [21-23]. RDH10 mutant mice display embryonic lethality at 13.5 d.p.c., and this is the earliest phenotype observed for a single retinol-metabolizing enzyme-deficient mouse [24]. However, it was suggested that endogenous RA synthesis initiates in the mouse at 7.5 d.p.c. $[2,25,26]$. Only double-mutant mice will clarify if enzymes with redundant functions are presents in the early embryonic development.

Among retinal oxidizing enzymes, RALDH2 is the only one expressed in undifferentiated ESC and its expression increases during EBs maturation (Figure 3C), reflecting activation of the RARE-Luc reporter gene (Figure $2 \mathrm{~A}$ ) and in vivo data. Indeed, in the mouse embryo RALDH2 is the first enzyme to appear during gastrulation at 7-7.5 d.p.c. $[27,28]$, and it is interesting to note that RALDH2 transcript was detected in all stages of bovine pre-attachment embryos, beginning from the oocyte through to the hatched blastocyst [29]. The two other RALDH enzymes (RALDH1 and RALDH3) appear later in development $[27,30]$. Thus, in EBs, resembling at 72 hours of differentiation the 7.5 d.p.c. embryo, the transcriptional activation of the genes involved in the retinoic acid synthesis pathway reflects the events observed in vivo.

Among the messenger RNAs for cytosolic retinoid binding proteins analyzed, only CRABP2 mRNA is up-regulated during EBs differentiation (Figure 3D). CRABP2 is a cytosol-to-nuclear shuttling protein, which facilitates RA transfer to the nucleus and binding to its receptor complex, suggesting that CRABP2 transcriptional increase could promote RA signaling. All the expression differences we observed between undifferentiated and differentiated ESC are due to their differentiation as EBs and not to the simple LIF withdrawal, as demonstrated by the values measured in ESC grown as monolayer without LIF for 4 days (ESC-LIF 4d). All together these data suggest 
A

B
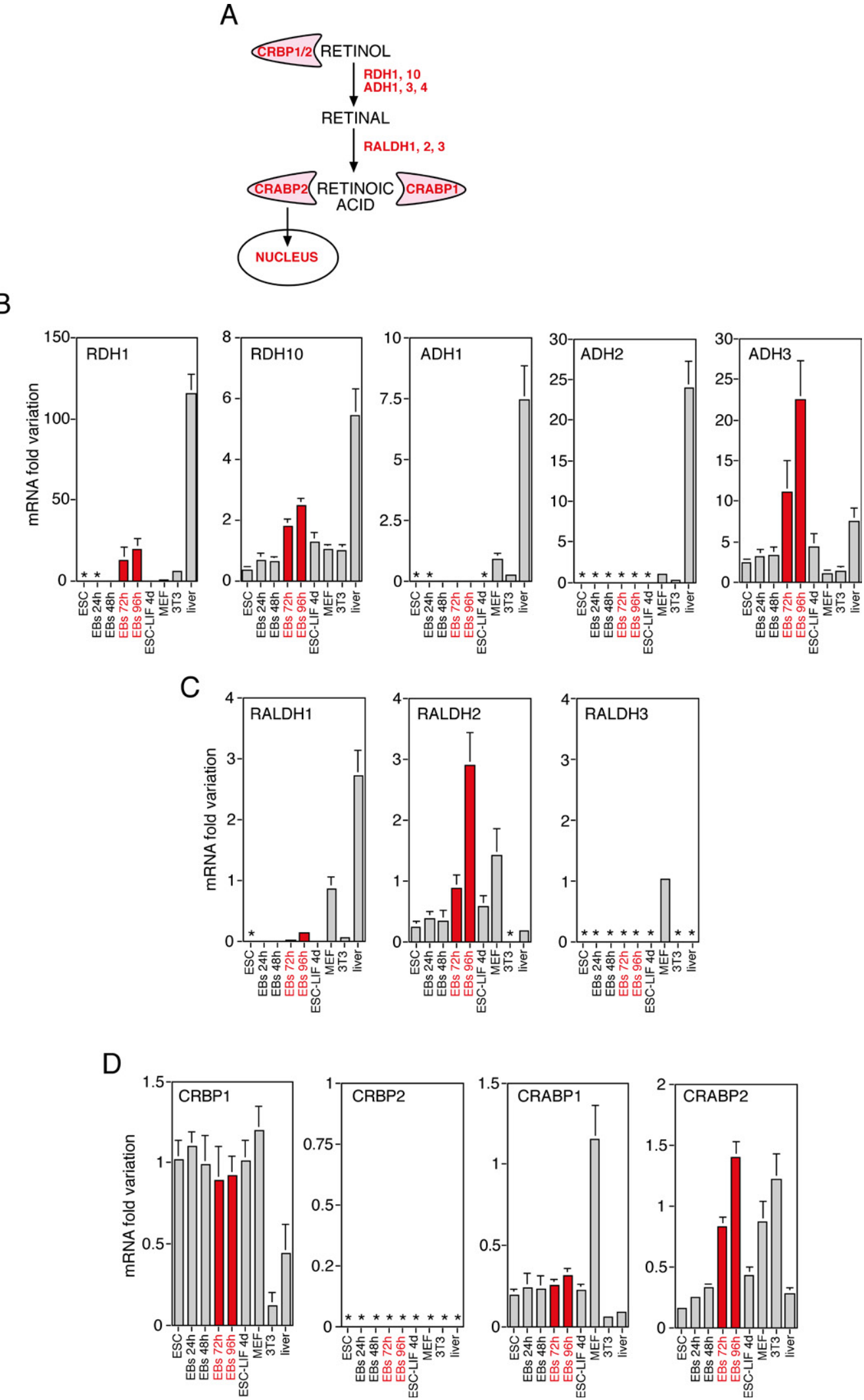

Figure 3: Expression analysis of RA biosynthetic pathway during ESC spontaneous differentiation. (A) Schematic view of the major components of the RA biosynthetic pathway. Expression of the indicated retinol-oxidizing enzymes (B), retinal-oxidizing enzymes (C), and cellular retinoid binding proteins (D) was evaluated by RT-qPCR analysis. Values are normalized to $\beta$-Actin expression and represented as fold variation compared with primary mouse embryonic fibroblasts (MEF). Asterisk indicates undetectable levels. Data represent average $\pm S D$ of three independent experiments. 
Citation: Neri F, De Clemente C, Orlandini M, Lentucci C, Anselmi F, et al. (2015) Murine Embryonic Stem Cells Synthesize Retinoic Acid to Promote their Own Differentiation. J Stem Cell Res Ther 5: 293. doi:10.4172/2157-7633.1000293

Page 6 of 8

that $\mathrm{RDH} 1, \mathrm{RDH} 10, \mathrm{ADH} 3, \mathrm{RALDH} 2$, and CRABP2 are responsible of the increase in RA synthesis and signalling in ESC during spontaneous differentiation as EBs.

\section{Identification of early differentially expressed genes in atRA treated ESC}

To identify direct atRA target genes in ESC, we analyzed the gene expression profile of E14 ESC treated with $1 \mu \mathrm{M}$ atRA for two hours. The analysis revealed that 176 genes were more than 2 -fold up-regulated and 96 more than 2-fold down-regulated (Figure 4A, GEO accession No. GSE66043). Among these, we found 22 TFs upregulated and 13 down-regulated (Figure $4 \mathrm{~B}$ ). Microarray data were independently validated by means of RT-qPCR analysis (Figure 4C). It is interesting to note that 3 TFs down-regulated (Otx2, Id 2 and Arid1a) are involved in ESC pluripotency maintaining [16,31,32], and 3 TFs up-regulated (Snail, Cdx1, Gata6) are known to be involved in pluripotency exit $[7,33,34]$. These data demonstrate the role of RA in promoting ESC differentiation acting directly on the regulation
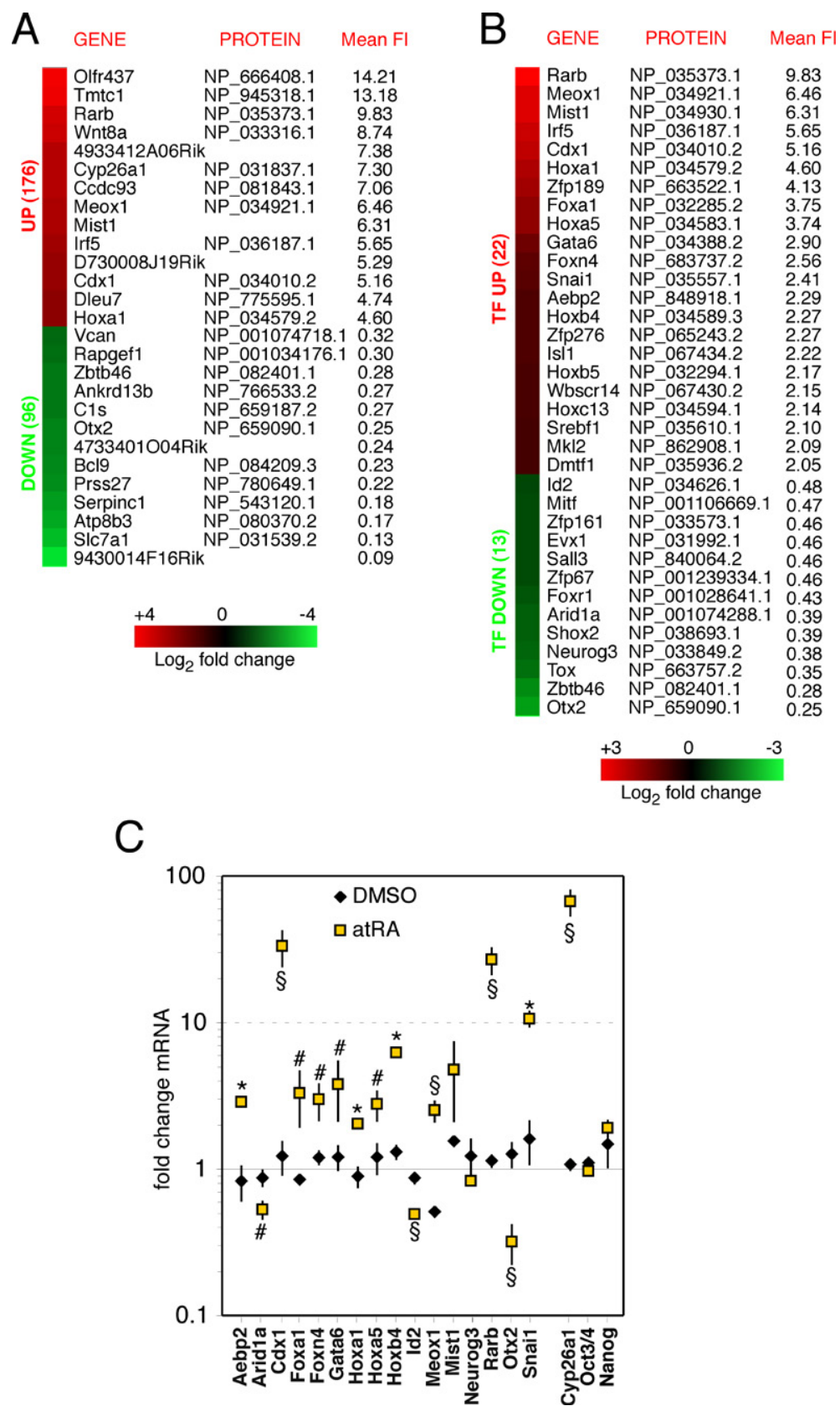

Figure 4: Identification of early differentially expressed genes in atRA treated ESC. (A) Heat map of microarray analysis showing the top up- and down-regulated genes following 2 hours atRA treatment (FC: Fold Change). (B) Heat map of microarray analysis showing the differentially expressed TFs. (C) The microarray data of 20 selected genes were validated by RT-qPCR analysis. Up-regulated genes are represented in red, down-regulated in green and negative controls (Nanog and Oct3/4) in black. Cyp26a1 was used as positive control. Gene expression was normalized to $\beta$-Actin expression and values obtained for DMSO or atRA treated cells are represented as fold change compared with corresponding values of untreated cells. Data represent average $\pm S D$ of three independent experiments *, $P<0.001$; $\S, \mathrm{P}<0.005 ; \#, \mathrm{P}<0.01(\mathrm{n}=3)$. 
of transcription factors involved in maintenance of, or exit from, pluripotency.

\section{Conclusions}

Cultivation and controlled differentiation of ESC has opened new frontiers both in regenerative medicine and biology of development. Many differentiation protocols use atRA to promote formation of primitive endoderm or all three germ layers. Here, we demonstrated that RA is synthesized by ESC during spontaneous differentiation as EBs and takes an active role to promote their own differentiation process. In Luciferase reporter gene activation assay, we observed that also undifferentiated ESC produce RA from retinol, even if at low levels. We suggest that this low RA synthesis helps to maintain ESC self-renewal as demonstrated by Wang et al. [35], and only higher levels of RA synthesized by differentiating EBs trigger the differentiation process.

In EBs, the transcriptional activation of the genes involved in the RA synthesis pathway reflects the events observed in vivo, with RDH1, $\mathrm{RDH} 10, \mathrm{ADH} 3$ and RALDH2 being the main enzymes providing RA for early organogenesis.

Microarray gene expression profile of ESC treated with atRA for two hours identified several TFs as direct target of RA receptors. Among them, Snail is one of the most up-regulated, confirming its important role in atRA-driven exit from the pluripotency. Cdx1 is also strongly up-regulared by atRA in ESC. In Xenopus, Cdx1 negatively regulates Oct3/4 during gastrulation, a step required for repression of pluripotency and germ layer differentiation [33]. Our data suggest that the axis atRA-Cdx1-Oct $3 / 4$ could be active also in murine or human ESC, but further experiments are necessary to confirm this hypothesis. This research is significant because it offers a comprehensive analysis of and demonstrates a role for endogenous RA synthesis during ESC cultivation and spontaneous differentiation. In the last years, a growing number of research groups turned to serum-free media for culturing and differentiating ESC. Therefore, our observations should be taken into account to evaluate in further comparative studies the effect of retinol supplementation of serum-free media.

\section{Acknowledgments}

This work was supported by Ministero Salute Bando cellule Staminali (grant number RF-TOS-2008-1226020)

\section{References}

1. Cunningham TJ, Duester G (2015) Mechanisms of retinoic acid signalling and its roles in organ and limb development. Nat Rev Mol Cell Biol 16: 110-123. [Pubmed]

2. Rossant J, Zirngibl R, Cado D, Shago M, Giguère V (1991) Expression of a retinoic acid response element-hsplacZ transgene defines specific domains of transcriptional activity during mouse embryogenesis. Genes Dev 5:1333-1344.

3. Niederreither K, Dollé $P$ (2008) Retinoic acid in development: towards an integrated view. Nat Rev Genet 9: 541-553. [Pubmed]

4. Capo-Chichi CD, Rula ME, Smedberg JL, Vanderveer L, Parmacek MS, et al. (2005) Perception of differentiation cues by GATA factors in primitive endoderm lineage determination of mouse embryonic stem cells. Dev Biol 286: 574-586. [Pubmed]

5. Torres JS Prieto J, Durupt FC, Broad S, Watt FM (2012) Efficient differentiation of embryonic stem cells into mesodermal precursors by BMP, retinoic acid and Notch signalling. PLoS One 7: e36405. [Pubmed]

6. Stavridis MP, Collins BJ, Storey KG (2010) Retinoic acid orchestrates fibroblast growth factor signalling to drive embryonic stem cell differentiation. Development 137: 881-890. [Pubmed]

7. Galvagni F, Lentucci C, Neri F, Dettori D, De Clemente C, et al. (2015) Snai1 promotes ESC exit from the pluripotency by direct repression of self-renewal genes. Stem Cells 33: 742-750. [Pubmed]

8. Neri F, Incarnato D, Krepelova A, Dettori D, Rapelli S, et al. (2015) TET1 is controlled by pluripotency-associated factors in ESCs and downmodulated by PRC2 in differentiated cells and tissues. Nucleic Acids Res. [Pubmed]

9. Gadue P, Huber TL, Paddison PJ, Keller GM (2006) Wnt and TGF-beta signaling are required for the induction of an in vitro model of primitive streak formation using embryonic stem cells. Proc Natl Acad Sci U S A 103: 1680616811. [Pubmed]

10. Orlandini M, Nucciotti S, Galvagni F, Bardelli M, Rocchigiani M, et al. (2008) Morphogenesis of human endothelial cells is inhibited by DAB2 via Src. FEBS Lett 582: 2542-2548. [Pubmed]

11. Neri F, Dettori D, Incarnato D, Krepelova A, Rapelli S, et al. (2014) TET1 is a tumour suppressor that inhibits colon ancer growth by derepressing inhibitors of the WNT pathway. Oncogene. [Pubmed]

12. Evellin S, Galvagni F, Zippo A, Neri F, Orlandini M, et al. (2013) FOSL1 controls the assembly of endothelial cells into capillary tubes by direct repression of $\hat{I} \pm \mathrm{v}$ and Î23 integrin transcription. Mol Cell Biol 33: 1198-1209. [Pubmed]

13. Incarnato D, Krepelova A, Neri F (2014) High-throughput single nucleotide variant discovery in E14 mouse embryonic stem cells provides a new reference genome assembly. Genomics 104: 121-127. [Pubmed]

14. Fehling HJ, Lacaud G, Kubo A, Kennedy M, Robertson S, at al. (2003) Tracking mesoderm induction and its specification to the hemangioblast during embryonic stem cell differentiation. Development 130:4217-4227.

15. Nostro MC, Cheng X, Keller GM, Gadue P (2008) Wnt, activin, and BMP signaling regulate distinct stages in the developmental pathway from embryonic stem cells to blood. Cell Stem Cell 2: 60-71. [Pubmed]

16. Zhang K, Li L, Huang C, Shen C, Tan F, et al. (2010) Distinct functions of BMP4 during different stages of mouse ES cell neural commitment. Development 137 2095-2105. [Pubmed]

17. Szondy Z, Reichert U, Bernardon JM, Michel S, Tóth R, et al. (1997) Induction of apoptosis by retinoids and retinoic acid receptor gamma-selective compounds in mouse thymocytes through a novel apoptosis pathway. Mol Pharmacol 51 972-982. [Pubmed]

18. Simandi Z, Balint BL, Poliska S, Ruhl R, Nagy L (2010) Activation of retinoic acid receptor signaling coordinates lineage commitment of spontaneously differentiating mouse embryonic stem cells in embryoid bodies. FEBS Lett 584: 3123-3130. [Pubmed]

19. Duester G, Farrés J, Felder MR, Holmes RS, Höög JO, et al. (1999) Recommended nomenclature for the vertebrate alcohol dehydrogenase gene family. Biochem Pharmacol 58: 389-395. [Pubmed]

20. Rout UK, Armant DR (2002) Expression of genes for alcohol and aldehyde metabolizing enzymes in mouse oocytes and preimplantation embryos. Reprod Toxicol 16: 253-258. [Pubmed]

21. Zhang M, Chen W, Smith SM, Napoli JL (2001) Molecular characterization of a mouse short chain dehydrogenase/reductase active with all-trans-retinol in intact cells, mRDH1. J Biol Chem 276: 44083-44090. [Pubmed]

22. Sandell LL, Sanderson BW, Moiseyev G, Johnson T, Mushegian A, et al. (2007) $\mathrm{RDH} 10$ is essential for synthesis of embryonic retinoic acid and is required for limb, craniofacial, and organ development. Genes Dev 21: 1113-1124. [Pubmed]

23. Cammas L, Romand R, Fraulob V, Mura C, Dollé P (2007) Expression of the murine retinol dehydrogenase 10 (Rdh10) gene correlates with many sites of retinoid signalling during embryogenesis and organ differentiation. Dev Dyn 236: 2899-2908. [Pubmed]

24. Kumar S, Sandell LL, Trainor PA, Koentgen F, Duester G (2012) Alcohol and aldehyde dehydrogenases: retinoid metabolic effects in mouse knockout models. Biochim Biophys Acta 1821: 198-205. [Pubmed]

25. Sirbu IO, Gresh L, Barra J, Duester G (2005) Shifting boundaries of retinoic acid activity control hindbrain segmental gene expression. Development 132: 2611-2622. [Pubmed]

26. Ang HL, Deltour L, Hayamizu TF, Zgombia-Knight M, Duester G (1996) Retinoic acid synthesis in mouse embryos during gastrulation and craniofacial development linked to class IV alcohol dehydrogenase gene expression. J Bio Chem 271: 9526-9534. [Pubmed] 
Citation: Neri F, De Clemente C, Orlandini M, Lentucci C, Anselmi F, et al. (2015) Murine Embryonic Stem Cells Synthesize Retinoic Acid to Promote their Own Differentiation. J Stem Cell Res Ther 5: 293. doi:10.4172/2157-7633.1000293

Page 8 of 8

27. Haselbeck RJ, Hoffmann I, Duester G (1999) Distinct functions for Aldh1 and Raldh2 in the control of ligand production for embryonic retinoid signaling pathways. Dev Genet 25: 353-364. [Pubmed]

28. Niederreither K, McCaffery P, Dräger UC, Chambon P, Dollé P (1997) Restricted expression and retinoic acid-induced downregulation of the retinaldehyde dehydrogenase type 2 (RALDH-2) gene during mouse development. Mech Dev 62: 67-78. [Pubmed]

29. Mohan M, Malayer JR, Geisert RD, Morgan GL (2002) Expression patterns of retinoid $X$ receptors, retinaldehyde dehydrogenase, and peroxisome proliferator activated receptor gamma in bovine preattachment embryos. Biol Reprod 66:692-700.

30. Clagett-Dame M, DeLuca HF (2002) The role of vitamin A in mammalian reproduction and embryonic development. Annu Rev Nutr 22: 347-381. [Pubmed]

31. Acampora D, Di Giovannantonio LG, Simeone A (2013) Otx2 is an intrinsic determinant of the embryonic stem cell state and is required for transition to a stable epiblast stem cell condition. Development 140: 43-55. [Pubmed]

32. Gao X, Tate P, Hu P, Tjian R, Skarnes WC, et al. (2008) ES cell pluripotency and germ-layer formation require the SWI/SNF chromatin remodeling component BAF250a. Proc Natl Acad Sci U S A 105: 6656-6661. [Pubmed]

33. Rousso SZ, Schyr RB, Gur M, Zouela N, Kot-Leibovich H, et al. (2011) Negative autoregulation of Oct $3 / 4$ through $\mathrm{Cdx} 1$ promotes the onset of gastrulation. Dev Dyn 240: 796-807. [Pubmed]

34. Fujikura J, Yamato E, Yonemura S, Hosoda K, Masui S, et al. (2002) Differentiation of embryonic stem cells is induced by GATA factors. Genes Dev 16: 784-789. [Pubmed]

35. Wang R, Liang J, Yu HM, Liang H, Shi YJ, et al. (2008) Retinoic acid maintains self-renewal of murine embryonic stem cells via a feedback mechanism. Differentiation 76: 931-945. [Pubmed] 